\title{
407 - The Baycrest Quick-Response Caregiver Tool: The Role for a New Tool for Caregivers of Persons with Dementia
}

Ken Schwartz, MDFRCPC, Robert Madan, MDFRCPC, Anna Berall, RN, Marsha Natadiria, BSC, Anna Santiago, MPH,MSC

Baycrest Health Sciences

\section{Background:}

Responsive behaviours in dementia are associated with poor outcomes for the person with dementia (PWD) and caregiver burnout. Family caregivers need a variety of tools to manage responsive behaviours. The Baycrest Quick-Response Caregiver Tool was developed to provide caregivers with a tool that can be used in real time. In this study, the feasibility, impact, and effectiveness of this new tool were studied in family caregivers and health care providers (HCP) using quantitative and qualitative measures.

Methods:

Family caregivers were recruited and were asked to complete a pre-survey before being sent the link to the educational tool. One month after the telephone survey, caregivers were sent an online post-survey to gather their feedback on the tool and the impact of the tool on caregiver well-being. Healthcare providers were also recruited and reviewed the tool through an online feedback survey. The feasibility, impact, and effectiveness of the tool were assessed using quantitative and qualitative measures.

Results:

Caregivers had a moderate degree of and reported a high level of competence - these scores were maintained throughout the study. Caregivers reported that tool positively impacted their compassion towards the person with dementia (PWD), and that their interactions with improved. $100 \%$ of HCP who completed the feedback survey would recommend the tool to other HCP and to caregivers of PWD. The caregivers and HCP provided specific suggestions for improvement.

Conclusions:

The Baycrest Quick-Response Caregiver Tool was found to be feasible and helpful. It provides caregivers and HCP with an additional approach for responsive behaviours. 